(1) $6-20-95$

12 copis
12

UCRL-ID-119073

\title{
Feasibility Study of a Microwave Radar System for Agricultural Inspection
}

\author{
Stephen Okelo-Odongo
}

October 3, 1994

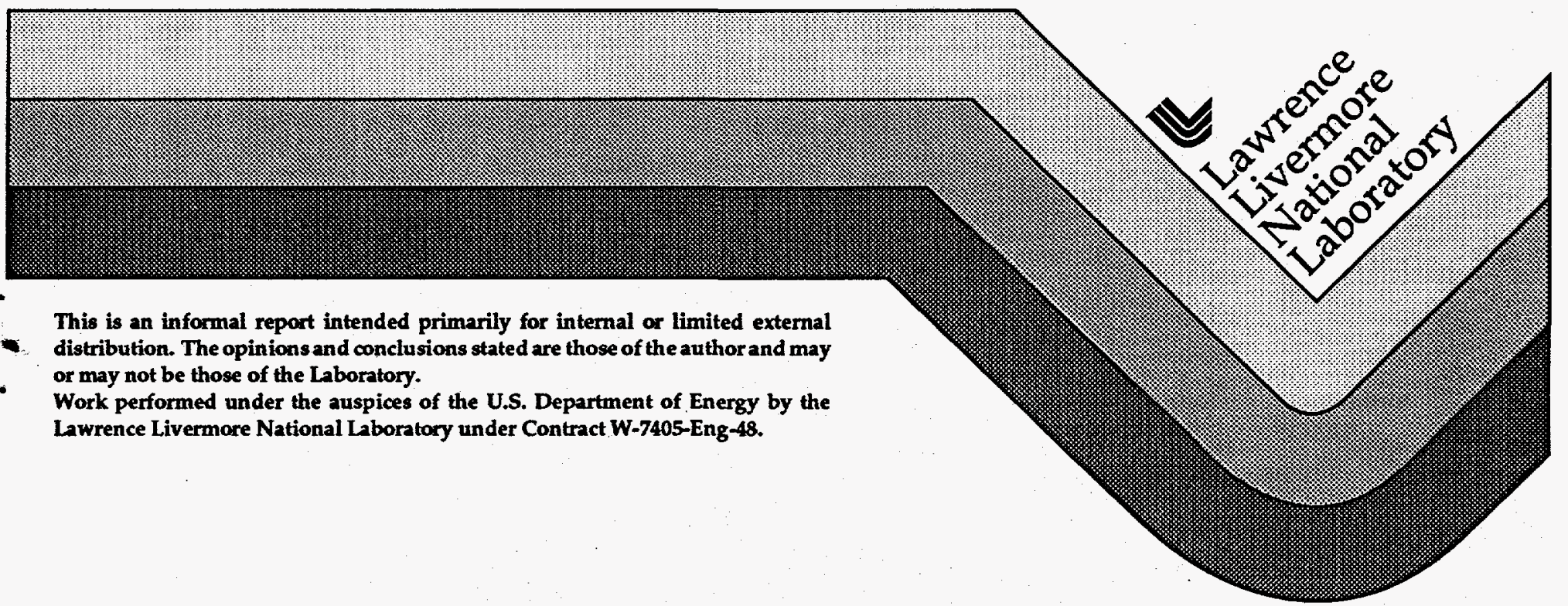




\section{DISCLAIMER}

This document was prepared as an account of work sponsored by an agency of the United States Government. Neither the United States Government nor the University of California nor any of their employees, makes any warranty, express or implied, or assumes any legal liability or responsibility for the accuracy, completeness, or usefulness of any information, apparatus, product, or process disclosed, or represents that its use would not infringe privately owned rights. Reference herein to any specific conmercial products, process, or service by trade name, trademark, manufacturer, or otherwise, does not necessarily constitute or imply its endorsement, recommendation, or favoring by the United States Government or the University of California. The views and opinions of authors expressed herein do not necessarily state or reflect those of the United States Government or the University of California, and shall not be used for advertising or product endorsement purposes.

This report has been reproduced directly from the best available copy.

Available to DOE and DOE contractors from the Office of Scientific and Technical Information

P.O. Box 62, Oak Ridge, TN 37831

Prices available from (615) 576-8401, FTS 626-8401

Available to the public from the

National Technical Information Service

US. Department of Commerce

5285 Port Royal Rd.

Springfield, VA 22161 


\section{DISCLAIMER}

Portions of this document may be illegible in electronic image products. Images are produced from the best available original document. 


\title{
Feasibility Study of a Microwave Radar System for Agricultural Inspection
}

\author{
A report prepared by DSED \\ Electronics Engineering, \\ 'Lawrence Livermore National Laboratory, \\ Livermore, California 94550
}

October 3, 1994

\section{Summary}

The feasibility of an impulse radar system for agricultural inspection is investigated. This system would be able to quickly determine the quality of foodstuffs that are passed through the system. A prototype was designed at the Lawrence Livermore National Laboratory and this report discusses it's evaluation. A variety of apples were used to test the system and preliminary data suggests that this technology holds promise for successful application on a large scale in food processing plants.

\subsection{Introduction}

A large portion of the cost of foodstuffs is a result of quality control performed at food processing plants. The quality of fruits and vegetables, for instance, is determined by visual inspection of the produce which is a time-consuming, tedious and subjective approach. This method is exacerbated by the fact that some defects take time to form and thus might not be visible during inspection but could develop fully whilst the fruit is in storage. Therefore a radar system might offer the possibility to detect defects in their early stages and thus offer a "go/no-go" analysis.

It is in this context that the Lawrence Livermore National Laboratory conducted an evaluation on the possible use of microwave radar technology to quickly qualify foodstuffs. The proof-of-concept system developed for this study was used to examine apples. The radar system launched a broadband pulse through the fruit and the resulting waveforms were digitized. As part of the analysis process a number of known defects were introduced to some apples. These apples were compared to a batch of control apples that were maintained for that purpose. Signal processing and statistical analysis were used to generate variance measurements of various features in the frequency spectrum of the apples. These results were used as the basis of the decision to either channel more resources to investigate this technology or to discontinue further research.

This report describes the experimental methodology that was used to determine the feasibility of a microwave radar based approach for quality control of fruits. Section 2 details some of the goals for the feasibility study. Section 3 explains the instrumentation and the experimental methodology used to accomplish the goals of the project. The results are listed in Section 4 and some problems to be wary of, in the event of more research, are examined in Section 5. The conclusion and suggestions for follow-on work are discussed in Section 6 . Also included is a selection of many of the key graphs generated during the project.

\subsection{Project Objectives}

The primary goal of this study was to determine if a microwave radar system could be used to distinguish desirable from undesirable apples. Undesirable apples were those affected by known and common defects (See Section 3.2). Secondary objectives were to obtain estimates for the dielectric constant (for "firmness") and the 
loss tangent (for moisture content) of the apples. Knowledge of the ideal geometry for the setup of the antenna system was also an important goal.

\subsection{Experimental Setup}

\subsection{Laboratory Equipment}

A pulser was used to generate a 400 ps FWHM gaussianlike pulse. This broadband pulse was used because the bandwidth of it's frequency spectrum extended from DC to the gigahertz $\left(10^{9} \mathrm{~Hz}\right)$ range. The hope of the study was that over this range there would be detectable changes in an apple's system response that could be attributed to a defect. The electromagnetic energy was launched through the fruit by a broadband dual ridged conical horn antenna. An identical receiver antenna was located across from the transmitting antenna with the polarizations aligned. The broadband dual ridged conical horn antennas were used in order to maintain pulse fidelity. Unfortunately, they acted as a high pass filter (with a cutoff frequency of about 1.5 $\mathrm{GHz}$ ) to the waveforms ${ }^{1}$. The receiver antenna was connected to a Tektronix ${ }^{\text {(i) }}$ CSA803 sampling oscilloscope that digitized the transmitted waveform. The waveforms thus obtained were transferred via a PC to a workstation for storage and processing.

Figure 1 illustrates the fast leading pulse of the signal received at the oscilloscope. The latter time ringing is due to the conical cutoff and the antenna-antenna interaction.

\subsection{Defect Simulation}

Twenty of each of the following types of apples were examined as part of the measurement series:

- Red Delicious

- Golden Delicious

- Granny Smith

- Fuji

Figure 1. Time domain waveform of the digitized pulse.

1. Time domain and frequency domain plots of the gaussian signal generated by the pulser as well as the frequency response of the hom antennas are in the Appendix.

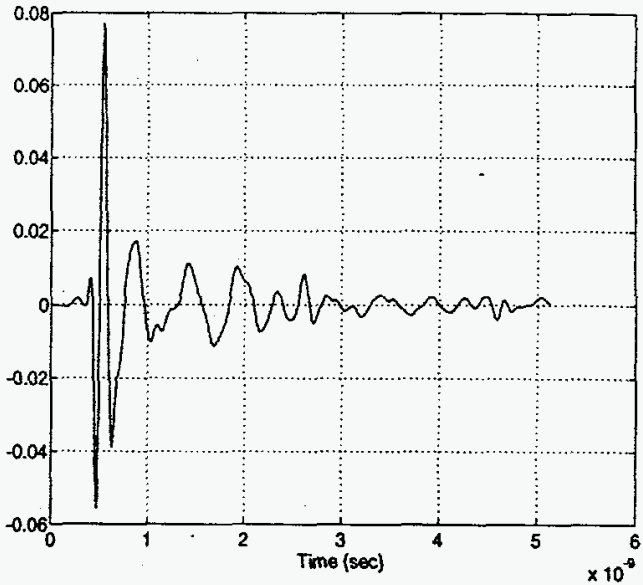

The following types of defects were introduced to the fruit:

- Room temperature spoilage

- Bruises

- Excess moisture pockets

- Worm hole cores

The apples were divided into sample sizes of four such that all types were represented equally in all defect simulations. The result of this distribution was that sixteen apples were subjected to each of the four defects and the remaining sixteen served as a control group. In each defect group of sixteen apples there were four Red Delicious, four Golden Delicious, four Granny Smith and four Fuji. The sample sizes in each group were made up of apples of the same type and were numbered 1 through 4.

The room temperature spoilage was modelled simply by allowing the apples to spoil at room temperature. Bruises were simulated by dropping the apples from predetermined heights onto a hard surface (a concrete floor) and were limited to one per apple. Care was taken not to break the skin of the apples. All the Number 1 bruise apples were dropped from a height of $10 \mathrm{~cm}$. The Number 2 bruise apples were dropped from $20 \mathrm{~cm}$ and so on for the others. Excess moisture pockets were simulated by injecting water with a syringe into the core or periphery of the apples. The bottoms of all Number 1 apples were injected with $0.5 \mathrm{cc}$ of water. The sides of apples Number 2 and Number 3 were mashed with the thumb to produce a bruise. Water was injected under the skin of these apples into the bruise $(0.3 \mathrm{cc}$ in Number 2 apples and $0.5 \mathrm{cc}$ in Number 3 apples). The tops of Number 4 apples were also 
mashed with the thumb and $1.0 \mathrm{cc}$ of water was injected into the resulting bruises. The worm holes were modelled by coring out small sections of apples using different size cores. A small core ( $1 \mathrm{~mm}$ diameter) and a large core (3 $\mathrm{mm}$ diameter) were used. The bottoms of Number 1 apples and the sides of Number 2 apples were cored out with the small core. The large core was used on the bottoms of Number 3 apples and the sides of the Number 4 apples.

The apples, with exception of the room temperature spoilage group, were kept in a refrigerator when not being measured. All the apples were measured on a daily basis (Monday-Friday).

\subsection{Orientation of Apples During Measurement}

The apples measured from Day 1 to Day 5 (Monday-Friday) were radiated with a horizontally polarized pulse and the horizontally polarized component of the corresponding transmitted wave was recorded (labelled "a" in the stored data set). The apples were radiated with the same side facing the horn then rotated 90 degrees about the axis of the stem for a new measurement (labelled " $b$ "). A third measurement was made with the apples on their side such that the wave "sliced" them from top to bottom (labelled "c").

In subsequent measurements (Day 9 through Day 15) the first recording was labelled " $x$ " and the apples were no longer rotated during the second recording. Also in the second measurement the vertical component of the transmitted wave was recorded instead of the horizontal (it was labeled " $y$ "). The third orientation was abandoned altogether.

\subsection{Results}

\subsection{Optimal Antenna Separation}

As too close an antenna-to-antenna spacing would result in pulse ringing and an unnecessarily large separation would result in poor coupling, the first task of the experiment was to determine the optimal spacing for good results.

This was accomplished by recording and comparing the signals obtained when the two antennas were separated by a distance varying from $16 \mathrm{~cm}$ to $26 \mathrm{~cm}$, measured in $2 \mathrm{~cm}$ increments. Another set of data was obtained from $25 \mathrm{~cm}$ to $50 \mathrm{~cm}$ measured in $5 \mathrm{~cm}$ increments. The results obtained revealed that ringing was not a significant issue with an antenna separation of about $20 \mathrm{~cm}$ or greater ${ }^{1}$.

Based on the findings above, the apples during all experiments were measured with an antenna separation of $30 \mathrm{~cm}$ and the center of the apples were halfway between the edges of each antenna. This separation was chosen to allow for a comfortable distance between the apple and the horns.

\subsection{Frequency Response Analysis}

Signal and systems theory was invoked in this study by designating the signal obtained with no apple between the horns as the input signal $x(t)$ and the signal obtained with an apple in between the horns as the output signal $y(t)$. Thus the apples were the "system" to be examined (see figure 2 below).

Figure 2. Apple as system $H(\omega)$

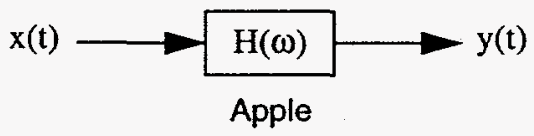

The system response of the apples was obtained by dividing the Fast Fourier Transform (FFT) of the output signal by the corresponding FFT of the input signal (Equation 1).

$$
H(\omega)=\frac{Y(\omega)}{X(\omega)}
$$

The effective bandwidth of the frequency response of the apples extended from about $2 \mathrm{GHz}$ to $10 \mathrm{GHz}$. Frequencies above $10 \mathrm{GHz}$ were discarded as the signal fell below noise levels whilst frequencies below $2 \mathrm{GHz}$ were ignored since they had been corrupted by the filtering effect of the antennas ${ }^{2}$. Figure 3 illustrates the typical spectrum of an apple's frequency response.

1. Plots illustrating this conclusion are in the Appendix

2. The Appendix contains the FFT of the signal $x(t)$ which clearly illustrates these facts. 
Figure 3. Typical apple's frequency response.

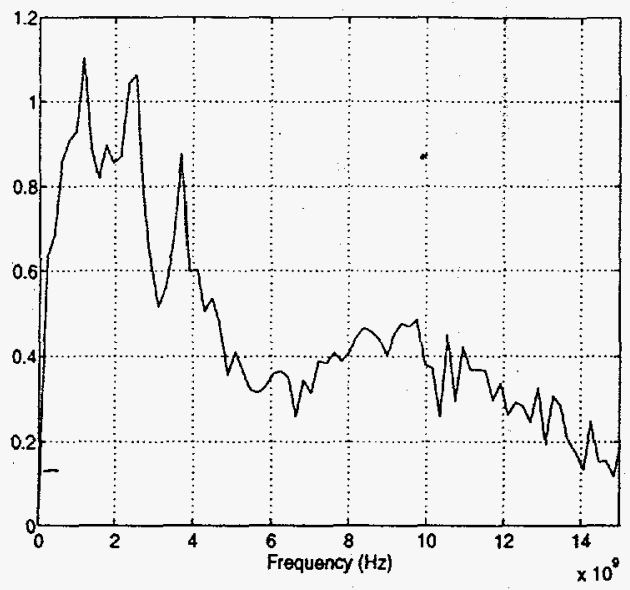

The first signals studied were those of the magnitude of the system response of the apples. Days 1 through 15 of apples Number 4 of the control group and apples Number 3 of the room temperature group were plotted. Trends in the data were sought that could serve as an aging metric. The plots suggested that the magnitude of the frequency response of the apples was not of significant usefulness in differentiating between apples.

As a result of this conclusion the phase response and the power spectrum of the apples was investigated. There was more variation in the phases of the apples but, as with the magnitude, there was no artifact observable that could serve to identify desirable from undesirable apples. The power spectrum was equally uninformative.

\subsection{Improved Data Set}

Due to the inconsistencies with the data recorded thus far (See Section 5.0) a new data set was created. Granny Smith apples were used (as control and bruise test apples). The apples were stored at room temperature and measurements were made on a MWF schedule for two weeks (this data was also stored according to the day of measurement). The first orientation of the apples used with the previous data set was chosen (horizontal to horizontal polarization).

The frequency spectrum (magnitude and phase) of these apples was scrutinized for differences between control and bruised apples. The results obtained with the previous data set were confirmed with this batch. Figures 4(a) and 4(b) below illustrate the lack of any clear data.

Figure 4(a) Phases of Control Apple

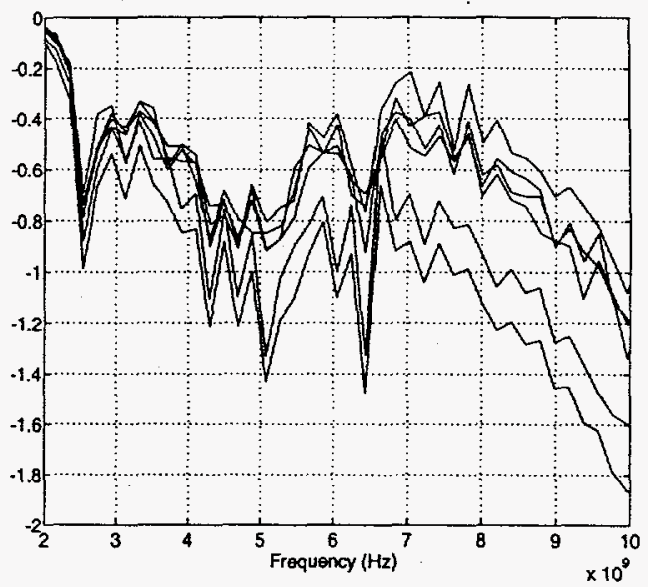

A new set of six recordings was made of the apples. They had always been measured with the same side facing the transmitting antenna. This orientation was labelled 0 degrees and was used as the first of the new set. The following five recordings were made by rotating the apples by 45 degrees (about the axis of the stem) before each measurement. The variance of the magnitude of the phase response of this data was calculated and plotted as a function of frequency. A first order approximation of the variance was also computed and displayed on the same graphs and served as the metric for distinguishing between the apples. 
Figure 4(b) Phases of Bruise Apple

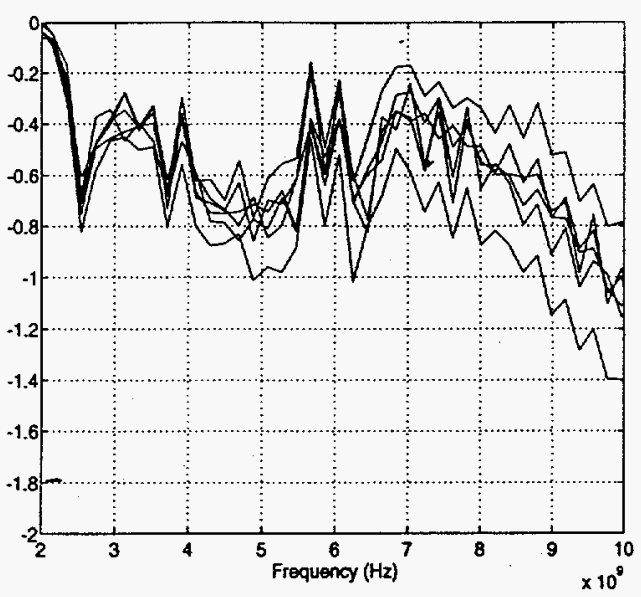

This information provided a definitive tool to differentiate the bruise apples from the control apples (see Figure 5). Some defective apples from the previous data set were tested as well as a bruised and unbruised pear and this method proved consistent in identifying the undesirable fruit. This measurement technique was an attempt to simulate a scanning array system that could be placed around the circumference of the test fruit.

In the figure below, the control apple is shown by the solid lines and the bruise apple is represented by the dashed line.

Figure 5. Variances of the Control and Bruise Apples and Their First Order Approximations.

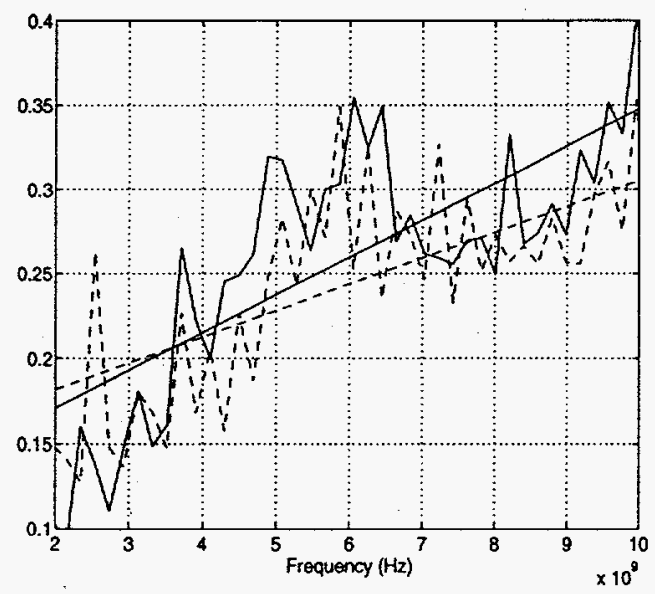

\subsection{Sources of Experimental Error}

\subsection{Inconsistent Recording}

Due to negligence, the measurements made from Day 1 to Day 5 were not recorded in the same manner as subsequent data ${ }^{1}$. This produced a greater resolution for later data and might have compromised the ability to accurately compare the differing data sets.

The most significant source of inconsistent data was that the separation between the horns was not fixed. The prototype system was simply a pulser connected to antennas which were placed on a non-conducting surface. This lack of accurate and consistent positioning of the antennas introduced phase shifts in the frequency domain of the data. This spacing and orientation issue still needs to be addressed for an optimized system.

\subsection{Ineffective Bruise Simulation}

Some of the apples used during the initial experiment (Day 1 through Day 15) had unmatured bruises which developed over time. In most cases these bruises were larger than the ones introduced into the apples as part of the study. Therefore the "bruise apple" set was not necessarily the only group of apples with bruises or even the most significant bruises.

\subsection{Conclusion}

It has been demonstrated that a microwave radar system can be used to distinguish desirable from undesirable apples in a fast and consistent manner. This was accomplished by calculating the first order approximation of the variance of the phase of the frequeuncy response of the apples. The defective apples (bruised in this case) seemed to display less variance which might have beeen due to fluids collecting in the bruise thus decreasing the dispersion of the wave propagating through the apple. More signal processing is still required to obtain a finer level of detail.

1. Section 7.1 contains plots illustrating the difference. 


\subsection{Suggestion For Follow-on Work}

A measurement system that can operate when the fruits are immersed in water should be investigated. This more closely resembles the type of transportation that the food processing industry currently employs and thus would require fewer modifications of their handling hardware. Follow-on work with other foodstuffs such as pears, peaches, kiwis, pomegranates or tomatoes is suggested.

\section{Acknowledgments}

The authors wish to thank the LLNL management, staff, and the GEM program for their indulgence, Kevin Smith of MTAC, and Yng Tau of Agritech for their comments and suggestions.

\subsection{Appendix}

\subsection{Selected Plots}

A number of figures illustrating various key plots are compiled in this section. There is no precise meaning attached to the vertical axis in any of the plots. Although it is a measure of voltage its exact value was not carried through in the study. Therefore it was used as an indication of "amplitude" to compare between similar plots.

Figure 6 illustrates the experimental setup from a systems theory perspective. The signal $g(t)$ is the gaussian pulse generated by the pulser which is then filtered by the horn antennas, $A(\omega)$. The next system, $H(\omega)$, and its corresponding input and output signals have been discussed in Section 4.2

Figure 6. System Representation of Experiment.

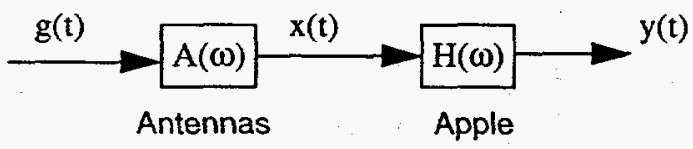

Figure 7 is a graph of the gaussian wave $g(t)$ generated by the pulse generator.
Figures $8(a)$ and $8(b)$ illustrate the magnitude and phase respectively of the frequency components of the gaussian wave $G(\omega)$ generated by the pulser.

Figure 7. Time domain plot of gaussian pulse $g(t)$.

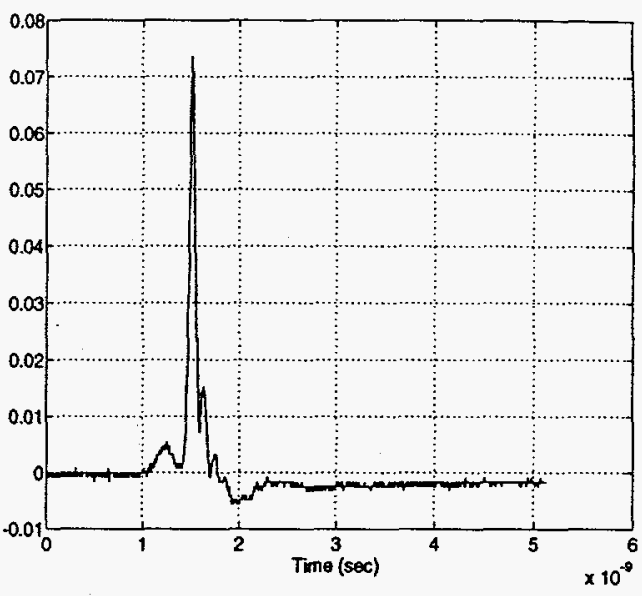

Figure 8(a) Magnitude of the FFT of the gaussian pulse.

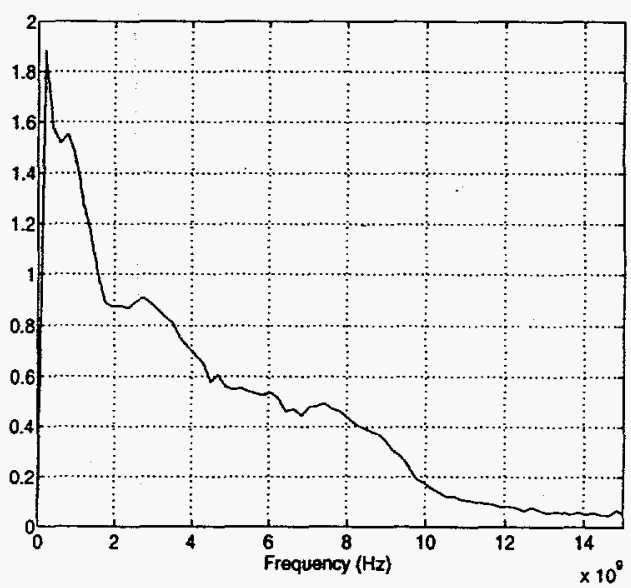


Figure 8(b) Phase of the FFT of the gaussian pulse.

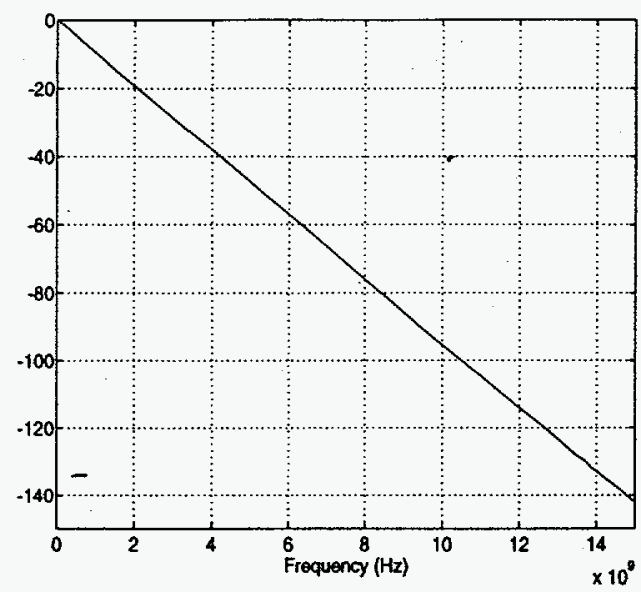

Figures 9 (a) and 9 (b) are the magnitude and phase respectively of the system response of the horn antennas $A(\omega)$.

Figure 9(a) Magnitude response of horn antennas $\mathrm{A}(\omega)$.

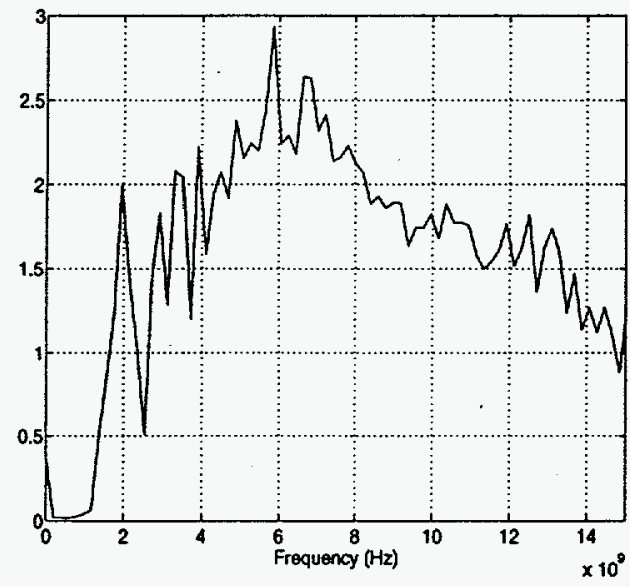


antenna interaction, ie, the wave reflected from the receiving horn to the transmitting horn and back.

Figure 11(a) Input Signal with $16 \mathrm{~cm}$ Horn Separation

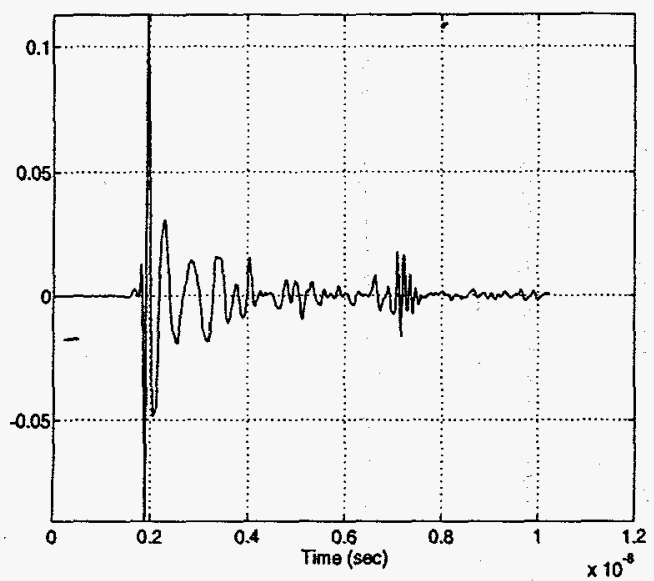

Figure 11(b) Input Signal with $20 \mathrm{~cm}$ Horn Separation

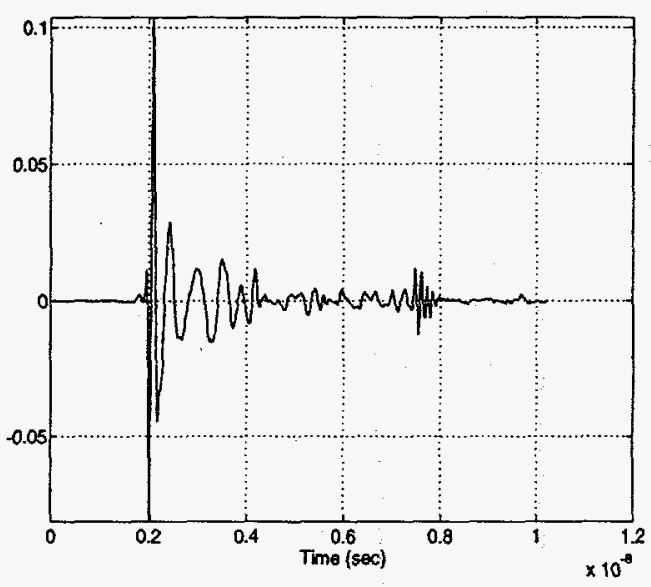

As the horn to horn distance is increased the the onset of the pulse is also shifted in time (hence the phase change in the frequency domain). The reflected pulse is moved farther away from the main pulse thus reducing ringing
Figure 11(c) Input Signal with $30 \mathrm{~cm}$ Horn Separation

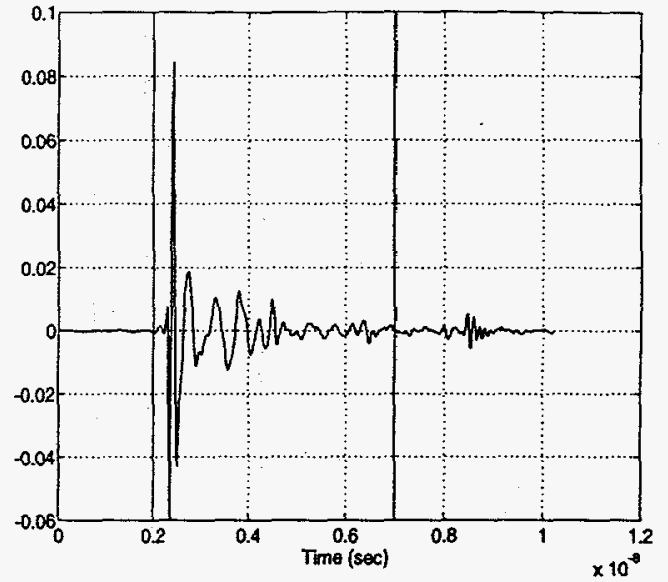

The plot above is similar to the data recorded from Day 1 to Day 5. The vertical lines indicate the portions of the signal that were extracted (see Section 7.3) to obtain a signal that more closely resembled the data recorded from Day 9 to Day 15.

The plot below further illustrates the time delay produced by a greater separation. In this figure the reflected pulse has moved completely off the digitized portion of the signal.

Figure 11(d) Input Signal with $50 \mathrm{~cm}$ Horn Separation

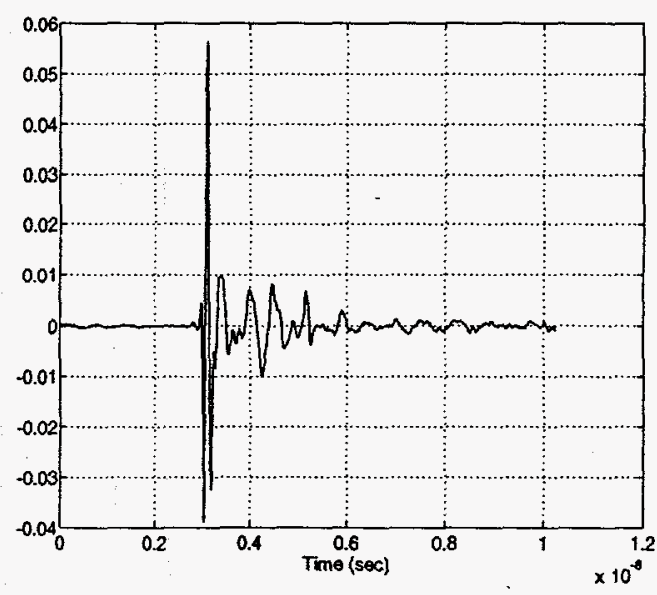

Figure 12(a) and 12(b) are the plots generated to confirm the validity of the variance method to distinguish the 
fruits. The first order approximation of the desirable fruit is shown as a solid line whilst the undesirable fruit is the dashed line.

\section{Figure 12(a) Variance for Old Data Set}

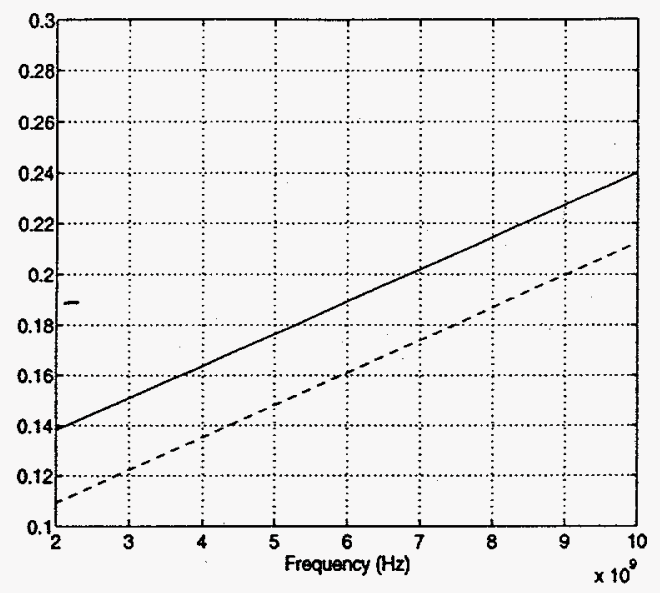

Figure 12(b) Variance for Pear

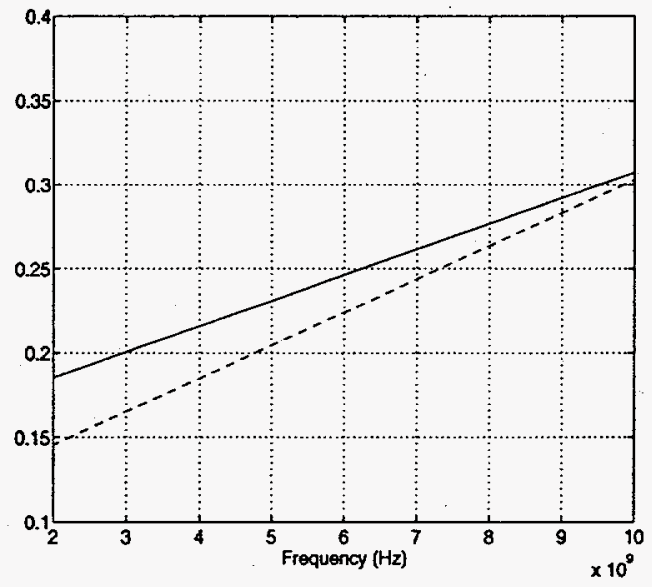

